\title{
RAS protein activator-like 1 is functionally involved in hypoxia resistance in breast cancer cells by targeting hypoxia inducible factor-1 $\alpha$
}

\author{
ZIMING HUANG $^{1}$, RONG WANG ${ }^{1}$, GANG WEI $^{1}$, RAN JIANG ${ }^{1}$, YUAN ZHENG ${ }^{1}$, \\ YIWEN TIAN $^{1}$, BO CHEN $^{1}$, CHUNMEI YE $^{1}$, MINGXING XUE $^{1}$ and CHUNJIAO YU ${ }^{2}$ \\ ${ }^{1}$ Department of Breast Surgery and ${ }^{2}$ Prenatal Diagnosis Center, Hubei Maternal \\ and Child Hospital, Wuhan, Hubei 430070, P.R. China
}

Received March 14, 2016; Accepted April 13, 2017

DOI: $10.3892 / \mathrm{ol} .2017 .6648$

\begin{abstract}
RAS protein activator-like 1 (RASAL1) is a member of the RAS GTPase-activating protein family, and previous studies indicate that RASAL1 is involved in the progression of hypoxia resistance in breast cancer cells. In the present study, increased levels of hypoxia inducible factor- $1 \alpha$ (HIF-1 $\alpha$ ) were observed to be accompanied with increased expression of RASAL1 in the breast cancer cell lines MCF-7 and MDA-MB-231 cells under hypoxia. Based on this, it was postulated that RASAL1 may serve a functional role in the development of hypoxia resistant in breast cancer cells. In the present study it was demonstrated that: i) Exogenous expression of RASAL1 in MCF-7 and MDA-MB-231 sensitized its reaction to the treatment of hypoxia, which is associated with its ability to directly reduce HIF- $1 \alpha$ expression, inhibit migration activity and decrease the accumulation of reactive oxygen species (ROS); ii) knockdown of RASAL1 reversed its reaction to treatment with hypoxia; iii) RASAL1 directly regulated the expression of HIF-1 $\alpha$ through the ROS-mediated, extracellular signal-regulated kinase and Akt pathway. These findings provide direct evidence that the RASAL1/HIF-1 $\alpha$ axis may serve an essential role in the hypoxia resistance of breast cancer cells, suggesting that this signaling cohort may serve as a novel therapeutic target for the treatment of breast cancer.
\end{abstract}

\section{Introduction}

Breast cancer is the most common cancer type and the leading cause of cancer-associated mortality in females worldwide (1).

Correspondence to: Dr Chunjiao Yu, Prenatal Diagnosis Center, Hubei Maternal and Child Hospital, 745 Wuluo Road, Wuhan, Hubei 430070, P.R. China

E-mail: chunjyu2008@163.com

Key words: RAS protein activator-like 1, hypoxia inducible factor-1 $\alpha$, breast cancer, reactive oxygen species, extracellular signal-regulated kinase, Akt
Developing an optimum strategy to overcome breast cancer is a major concern. Tumor hypoxia is the main cause of treatment failure in a variety of malignancies (2). Mounting evidence has been gathered as a result of identifying genes inducing hypoxia resistance in the past decade, particularly hypoxia inducible factor- $1 \alpha(\mathrm{HIF}-1 \alpha)$, which is activated in regions of intratumoral hypoxia, and mediates angiogenesis, invasion, metastasis and resistance to radiation therapy and chemotherapy (3). However, the link between HIF-1 $\alpha$ and hypoxia resistance in breast cancer therapy remains unclear.

RAS proteins control numerous cellular processes, including cell migration, proliferation, differentiation and survival. The RAS GTPase-activating protein gene RAS protein activator-like 1 (RASAL1) has been demonstrated to be a tumor suppressor gene which acts as a negative modulator of the RAS signaling pathway by catalyzing RAS inactivation. RASAL1 expression is decreased in numerous tumors, including colorectal tumors (4), thyroid cancer (5), gastric cancer (6), prostate cancer and bladder cancer (7). However, to date, there are no reports of it being associated with breast cancer. RASAL1 is stimulated by increases in intracellular $\mathrm{Ca}^{2+}$ leading to the attenuation of RAS activation and mitogen-activated protein kinase (MAPK) activity, and contributing to tumor progression through its weakened anti-RAS activity. The most significant sources of cellular stress in breast cancer are nutrient deprivation, hypoxia and the intracellular accumulation of calcium ions; hypoxia-induced intracellular calcium overload activates the RASAL1 gene and contributes to its tumor suppressor effect.

Intratumoral hypoxia and genetic alterations may lead to HIF-1 $\alpha$ overexpression, which has been associated with increased mortality in breast cancer $(8,9)$. Under hypoxic conditions, reactive oxygen species (ROS) increase significantly. The abnormal ROS accumulation activates the phosphoinositide 3-kinase/Akt and MAPK/extracellular signal-regulated kinase (ERK) pathways, which have been considered as the crucial regulatory pathways in carcinogenesis and tumor angiogenesis, and activators of HIF-1 $\alpha$. Based on findings that hypoxia functionally regulates the expression of RASAL1 and the significance of HIF-1 $\alpha$ in cancer cell intratumoral hypoxia, it is postulated that RASAL1 may serve 
a role in the hypoxia resistance of breast cancer cells, which may be mediated by its regulation of HIF-1 $\alpha$ expression (10). The aim of the present study was to explore the functional significance of the RASAL1/HIF-1 $\alpha$ axis in hypoxia resistance in breast cancer cells, and the findings suggest that RASAL1 may promote hypoxia resistance in breast cancer, mediated via its regulation of HIF-1 $\alpha$. RASAL1 may be a novel therapeutic target for breast cancer therapy.

\section{Materials and methods}

Cell culture and hypoxia treatment. The human breast cancer cell line MCF-7 was cultured in modified Eagle's medium (MEM; Thermo Fisher Scientific, Inc., Waltham, MA, USA), and MDA-MB-231 was cultured in L-15 medium (Gibco; Thermo Fisher Scientific, Inc.) supplemented with $10 \%$ fetal bovine serum (FBS; Thermo Fisher Scientific, Inc.), $100 \mathrm{U} / \mathrm{ml}$ penicillin and $100 \mathrm{U} / \mathrm{ml}$ streptomycin. Cells were maintained in a humidified incubator at $37^{\circ} \mathrm{C}$ with $5 \% \mathrm{CO}_{2}$. Hypoxic culture conditions $\left(1 \% \mathrm{O}_{2}\right)$ were achieved in a custom-designed hypoxic incubator via a continuous infusion of a pre-analyzed gas mixture $(95 \%$ $\mathrm{N}_{2}$ and $5 \%\left(\mathrm{CO}_{2}\right.$ ). Media was serum-starved in MEM or L-15 medium plus $0.5 \% \mathrm{FBS}$ at least $8 \mathrm{~h}$ before hypoxic culture.

Plasmid and cell transfection. For RASAL1 overexpression experiments, RASAL1 expression plasmid was purchased from BioVector NTCC, Inc. (BC093724; Beijing, China). MCF-7 and MDA-MB-231 cells were transfected using Lipofectamine ${ }^{\circledR} 3000$ reagent (Thermo Fisher Scientific, Inc.) using $1 \mu \mathrm{g} /$ well plasmid in a six-well plate following the manufacturer's protocol. Forty-eight $h$ after transfection, the cells were harvested for cell proliferation assay, invasion assay, western blot assay and real-time quantitative polymerase chain reaction (qPCR) assay.

For RASAL1 knockdown experiments, short hairpin RASAL1 (shRASAL1) and scramble were generated as previously described (4), and lentivirus was produced in transfected 293FT packaging cells. The cells were harvested for cell proliferation assay, invasion assay, western blot assay and qPCR assay $48 \mathrm{~h}$ after infection.

MTT assay. A 100- $\mu 1$ suspension of MCF-7 and MDA-MB-231 cells was seeded on 96-well plates with or without hypoxia conditions for various times $(0,24,48$ and $72 \mathrm{~h})$ at a density of $5 \times 10^{4}$ cells per well. Following incubation for the designated period of time, MTT was added to each well at a final concentration of $0.5 \mathrm{mg} / \mathrm{ml}$ for $4 \mathrm{~h}$, and the resulting formazan crystals were dissolved in dimethyl sulfoxide. Optical density was measured at $490 \mathrm{~nm}$ with background subtraction at $630 \mathrm{~nm}$ using a plate microreader (MK3; Thermo Fisher Scientific, Inc.). The growth inhibitory ratio was calculated.

Western blot analysis. Thirty micrograms of cell lysate and tumor tissue lysate were separated on $12 \%$ sodium dodecyl sulfate-polyacrylamide gel electrophoresis gels and then transferred onto nitrocellulose membranes. Specific monoclonal anti-RASAL1 (ab170711) and monoclonal anti-HIF-1 $\alpha$ (ab113642) primary antibodies (Abcam Biotechnology, Cambridge, MA, USA) were used, and anti-Phospho-ERK1/2 (Thr202/Tyr204; cat. no. 4730), anti-ERK1/2 (cat. no. 9102),
anti-Phospho-Akt (Ser473; cat. no. 4060), anti-Akt (cat. no. 9272) and horseradish peroxidase-conjugated immunoglobulin were used as secondary antibodies (Cell Signaling Technology, Inc., Danvers, MA, USA). West Pico chemiluminescent substrate (Pierce; Thermo Fisher Scientific, Inc.) was used to visualize protein bands, which were quantified using densitometry image analysis software (Image Master VDS; Pharmacia Biotech, Uppsala, Sweden). Normalization was made against $\beta$-actin (ab6276) expression.

qPCR analysis. Total RNA was extracted from cultured cells using TRIzol ${ }^{\circledR}$ reagent (Invitrogen; Thermo Fisher Scientific, Inc.). DNaseI-treated RNA was used for first-strand cDNA synthesis using M-MLV reverse transcriptase (Promega Corporation, Madison, WI, USA) and oligo (dT) 15 according to the manufacturer's protocol and $1 \mu \mathrm{l}$ cDNA samples were used for conventional PCR amplifications. qPCR analysis was performed in a real-time PCR system (StepOne, Applied Biosystems; Thermo Fisher Scientific, Inc.), and the expression levels of RASAL1 and HIF-1 $\alpha$ were normalized to GAPDH determined by a SYBR-Green-based comparative cycle threshold method. The qPCR primers were: RASAL1-F: 5'-TGGATTTCTCTTCTTGCGATTCT-3', RASAL1-R: 5'-TGTTGGTCCCGAAGGTCAA-3'; HIF-1 $\alpha-F$ : 5'-ACTTGG CAACCTTGGATTGGA-3', HIF-1 $\alpha-\mathrm{R}: 5^{\prime}$-ATCTCCGTCCCT CAACCTCT-3'; GAPDH-F: 5'-TGGGATTTCCATTGATGA CAAG-3', GAPDH-R: 5'-CCACCCATGGCAAATTCC-3'.

In vitro invasion assay. Invasion of the breast cancer cell lines was examined in a modified two-chamber assay as described previously (11). Cells $\left(2 \times 10^{5}\right)$ were seeded on the upper side of six-well Transwell plates (Costar, Cambridge, MA, USA) coated with Matrigel (BD Biosciences, Heidelberg, Germany) diluted at a 1:2 ratio with medium and incubated for $24 \mathrm{~h}$. The lower chamber was filled with Dulbecco's modified Eagle's medium (DMEM) containing 10\% FBS. Following incubation, cells on the upper side of the membrane were wiped off and the membrane was fixed with $4 \%$ paraformaldehyde and $0.25 \%$ glutaraldehyde. Cells on the lower side of the membrane were stained with $0.5 \%$ methylene blue in $50 \%$ methanol and counted.

Measurement of intracellular ROS accumulation. Intracellular ROS accumulation was estimated by flow cytometry (FCM) using the dichloro-dihydro-fluorescein diacetate (DCFH-DA) fluorescent probe. Briefly, the treated cells were collected and washed twice with MEM or L-15 medium. The level of intracellular ROS was determined by incubating the cells with the DCFH-DA working solution $(25 \mu \mathrm{mol} / \mathrm{l})$ at $37^{\circ} \mathrm{C}$ for $30 \mathrm{~min}$. Following incubation, cells were washed twice with MEM or L-15 medium and then analyzed using FCM to determine the DCF fluorescence intensity at excitation and emission wavelengths of 488 and $525 \mathrm{~nm}$, respectively.

Plasmid construction and luciferase assay. The entire human HIF-1 $\alpha$ 3'-untranslated region (UTR) segment was amplified by PCR using human genomic DNA as a template. The PCR products were inserted into the pGL3-Basic plasmid (Ambion; Thermo Fisher Scientific, Inc.). Efficient insertion was confirmed by sequencing. For luciferase reporter assays, $1 \mu \mathrm{g}$ firefly luciferase reporter plasmid, $0.1 \mu \mathrm{g} \beta$-galactosidase 

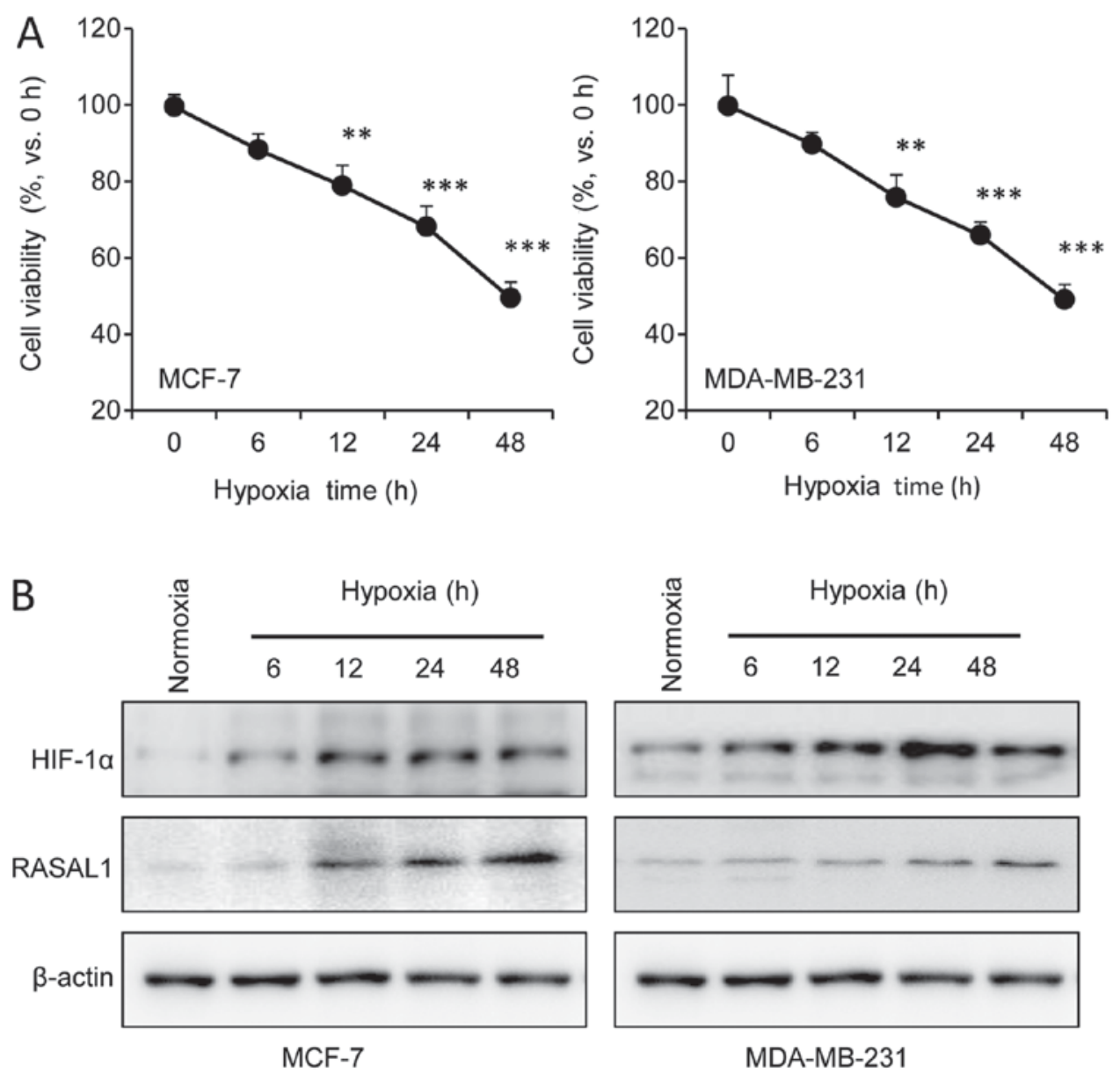

Figure 1. Hypoxia increases the expression of RASAL1 and HIF-1 $\alpha$ in MCF-7 and MDA-MB-231 cells. (A) MCF-7 and MDA-MB-231 cells were treated with hypoxia for various times, and cell viability was analyzed with an MTT assay. (B) Western blot analysis of HIF-1 $\alpha$ and RASAL1 in MCF-7 and MDA-MB-231 cells. Total lysates were prepared from cells treated with hypoxia for the indicated times. Antibodies against HIF-1 $\alpha$ and RASAL1 were used in this assay. Anti- $\beta$-actin was used as the protein loading control. Each bar represents the mean \pm standard error of the mean. The results were reproduced in three independent experiments. ${ }^{* *} \mathrm{P}<0.01,{ }^{* * *} \mathrm{P}<0.001$ vs. control group. RASAL1, RAS protein activator-like 1 ; HIF-1 $\alpha$, hypoxia inducible factor- $1 \alpha$.

expression vector (Ambion) and $0.3 \mu \mathrm{g}$ RASAL1 and control plasmid were transfected into cells in six-well plates with Lipofectamine ${ }^{\circledR} 3000$. $\beta$-galactosidase vector was used as a transfection control. At $24 \mathrm{~h}$ after transfection, $10 \mathrm{mmol} / \mathrm{l}$ $\mathrm{N}$-acetyl cysteine (NAC) was added to treat the cells for another $36 \mathrm{~h}$, and finally cells were assayed using luciferase assay kits (E1910, Promega Corporation).

Statistical analysis. Results are expressed as the means \pm standard error of the mean from at least three independent experiments. Significance analysis was performed using a two-tailed Student's t-test, and $\mathrm{P}<0.05$ and $\mathrm{P}<0.01$ were considered to indicate a significant and highly significant difference, respectively.

\section{Results}

Hypoxia increases the expression of RASAL1 and HIF-1a in MCF-7 and MDA-MB-231 cells. Following exposure to hypoxia for various times, MCF-7 and MDA-MB-231 cells demonstrated a time-dependent decrease in cell viability based on the MTT assay. Compared with the normoxic condition, the cell viability significantly decreased under hypoxic conditions for $>12 \mathrm{~h}$, indicating that the hypoxic injury model was successful (Fig. 1A). The protein expression of HIF-1 $\alpha$ and RASAL1 gradually increased in a time-dependent manner, as demonstrated by western blot assay (Fig. 1B). Combined data from the two cell lines revealed that the expression of HIF-1 $\alpha$ was correlated with RASAL1 expression under hypoxia.

Overexpression of RASAL1 in breast cancer cell lines inhibits hypoxic cell proliferation and cell invasion in vitro by decreasing protein levels of HIF-1 $\alpha$ and intracellular ROS accumulation. To assess the effect of RASAL1 on cell proliferation and invasion ability under hypoxia in breast cancer cells, MCF-7 and MDA-MB-231 cells were transfected with cDNA encoding the complete coding region of human RASAL1 using Lipofectamine ${ }^{\circledR} 3000$, then cultured under hypoxia for $24 \mathrm{~h}$. The two groups demonstrated overexpression at the protein and mRNA levels (Fig. 2A). RASAL1 overexpression in MCF-7 and MDA-MB-231 cells respectively demonstrated a 42 and $75 \%$ increase in the cell viability rate compared with the vector control and parental groups following hypoxia treatment for $24 \mathrm{~h}$ (Fig. 2B). The impact of RASAL1 on cell invasion was also observed in vitro; RASAL1 induced an increase in the invasion rate compared with the vector control and parental groups (Fig. 2C). The DCFH-DA fluorescent probe was used for measuring intracellular ROS levels, and 
A
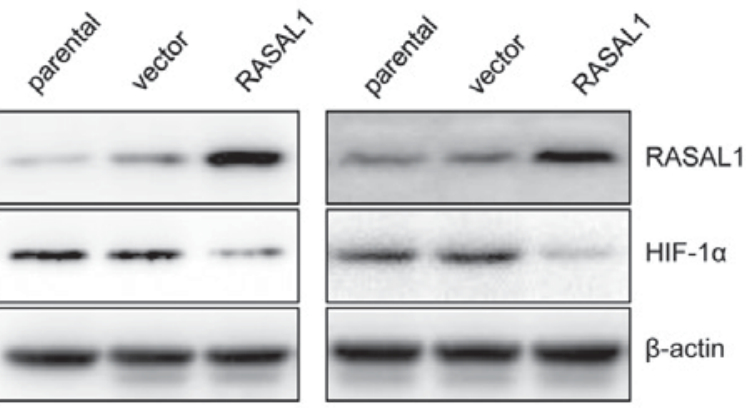

MCF-7

MDA-MB-231
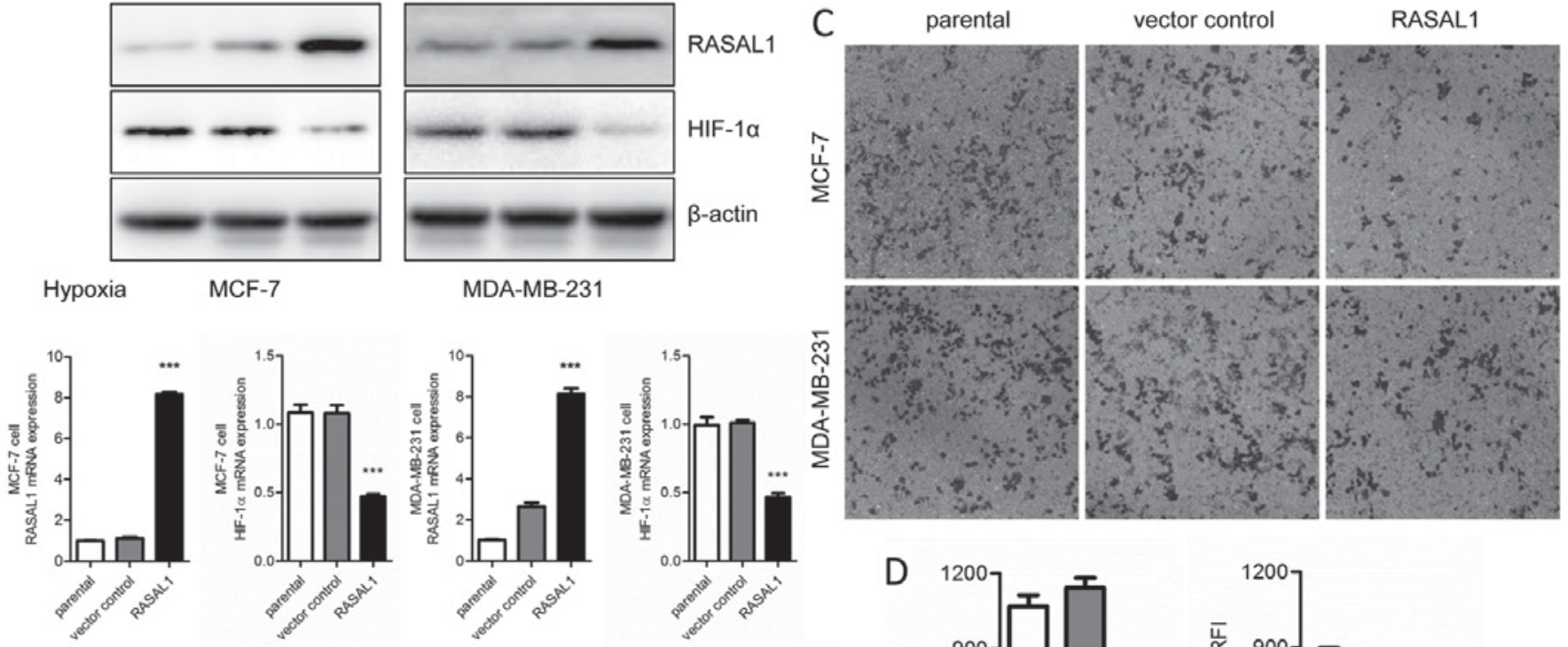

B
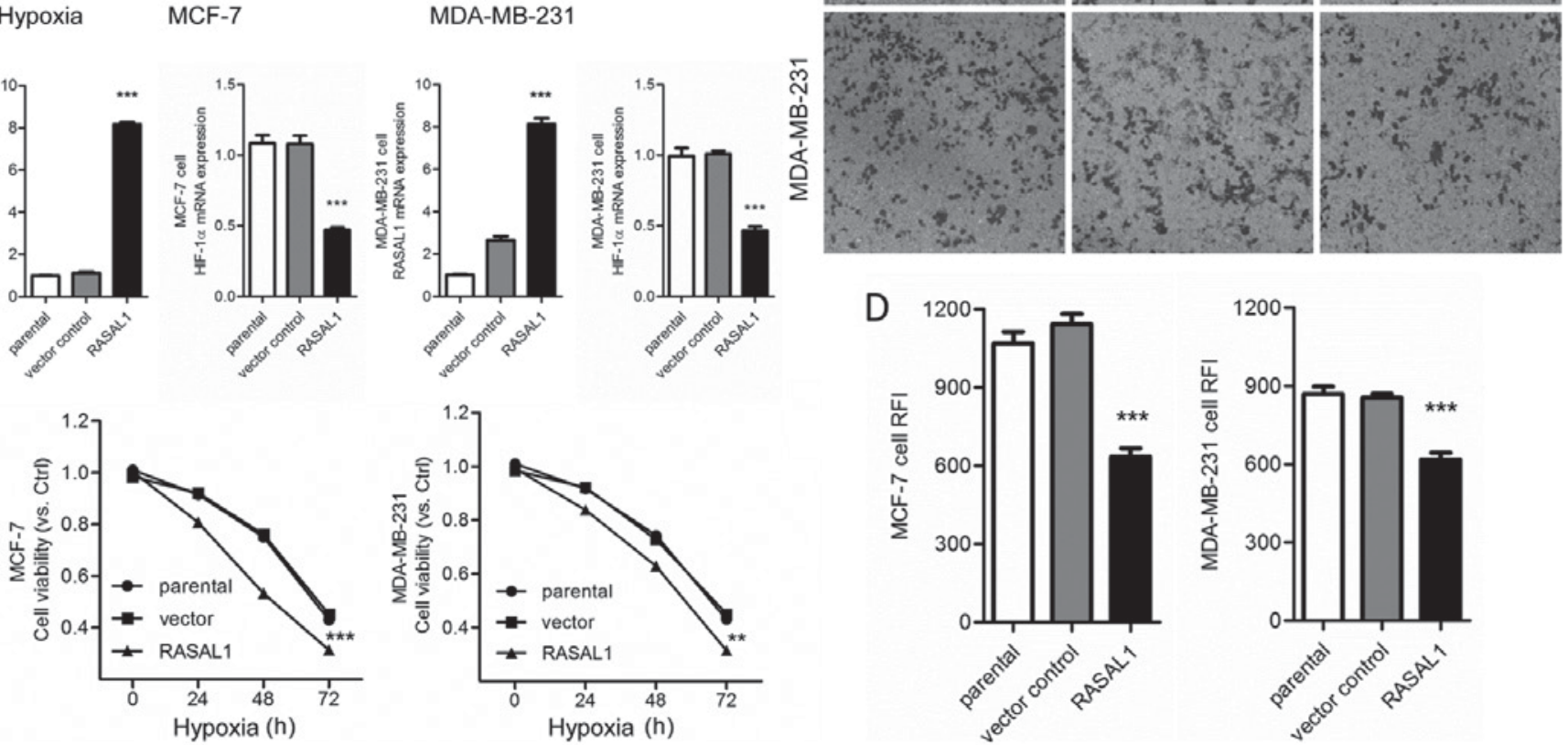

Figure 2. Overexpression of RASAL1 in breast cancer cell lines inhibits hypoxic cell proliferation and cell invasion in vitro by decreasing the protein levels of HIF-1 $\alpha$ and intracellular ROS accumulation. (A) MCF-7 and MDA-MB-231 cells were transfected with RASAL1 cDNA. RASAL1 and HIF-1 $\alpha$ levels were assessed by quantitative polymerase chain reaction and western blot analysis $48 \mathrm{~h}$ after transfection. (B) Cell viability is demonstrated as the fold change in viability from the normoxic control in MCF-7 and MDA-MB-231 cells treated with hypoxia for various times. (C) Repression of invasion ability by RASAL1 in MCF-7 and MDA-MB-231 cells under hypoxia for $24 \mathrm{~h}$ was detected using a Transwell assay (magnification, $\mathrm{x} 100$ ). (D) Intracellular ROS accumulation was detected using a dichloro-dihydro-fluorescein diacetate probe after $24 \mathrm{~h}$ of hypoxia treatment. Each bar represents the mean \pm standard error of the mean. The results were reproduced in three independent experiments. ${ }^{* *} \mathrm{P}<0.01,{ }^{* * * *} \mathrm{P}<0.001$ vs. the control group. RASAL1, RAS protein activator-like $1 ;$ HIF-1 $\alpha$, hypoxia inducible factor- $1 \alpha$; ROS, reactive oxygen species; RFI, relative fluorescence intensity.

decreased ROS levels were observed under hypoxic conditions in the group with RASAL1 overexpression (Fig. 2D).

Knockdown of RASAL1 in breast cancer cell lines ameliorates hypoxic cell viability and cell invasion in vitro by increasing HIF-1 $\alpha$ protein levels and intracellular ROS levels. Next, the effect of RASAL1 was tested on cell viability and invasion ability in MCF-7 and MDA-MB-231 cells transduced with either scrambled or shRASAL1 lentivirus. Transduction of MCF-7 and MDA-MB-231 cells with shRASAL1 lentiviral particles decreased RASAL1 at the protein and mRNA level (Fig. 3A). RASAL1 knockdown MCF-7 and MDA-MB-231 cells demonstrated a significant increase in the cell viability rate compared with the scramble and parental groups following hypoxia treatment for $72 \mathrm{~h}$ (Fig. 3B). shRASAL1 lentivirus induced an increased invasion rate compared with the vector control and parental groups (Fig. $3 \mathrm{C}$ ), as well as increasing intracellular ROS levels (Fig. 3D).

RASAL1 regulates HIF-1 $\alpha$ expression through the ROS-ERK/Akt pathway. RASAL1 expression has been reported to be associated with ROS generation, and increased ROS production activates the ERK and Akt pathways, and finally upregulates key proangiogenic molecule HIF-1 $\alpha$ (12). Therefore, it was next assessed whether RASAL1 affected the expression of HIF-1 $\alpha$ through a ROS-dependent pathway. As shown in Fig. 4A, decreased expression of RASAL1 enhanced ROS-stimulated phosphorylation of ERK1/2 and Akt in MCF-7 and MDA-MB-231 cells under hypoxic conditions. In addition, dual luciferase assay revealed that inhibited transduction of RASAL1 in MCF-7 and MDA-MB-231 cells increased the gene promoter luciferase activity of HIF-1 $\alpha$ 3'-UTR (Fig. 4B). Additionally, treatment with $10 \mathrm{mmol} / \mathrm{l} \mathrm{NAC}$ for $36 \mathrm{~h}$ significantly inhibited the phosphorylation of Akt and ERK1/2 and the transactivation of HIF-1 $\alpha$ gene promoters. These findings provide evidence that RASAL1 may directly regulate the expression of HIF-1 $\alpha$ through the ROS-mediated ERK and Akt pathways.

\section{Discussion}

Intratumoral hypoxia has long been considered a driving force of tumor progression and a negative prognostic factor in 


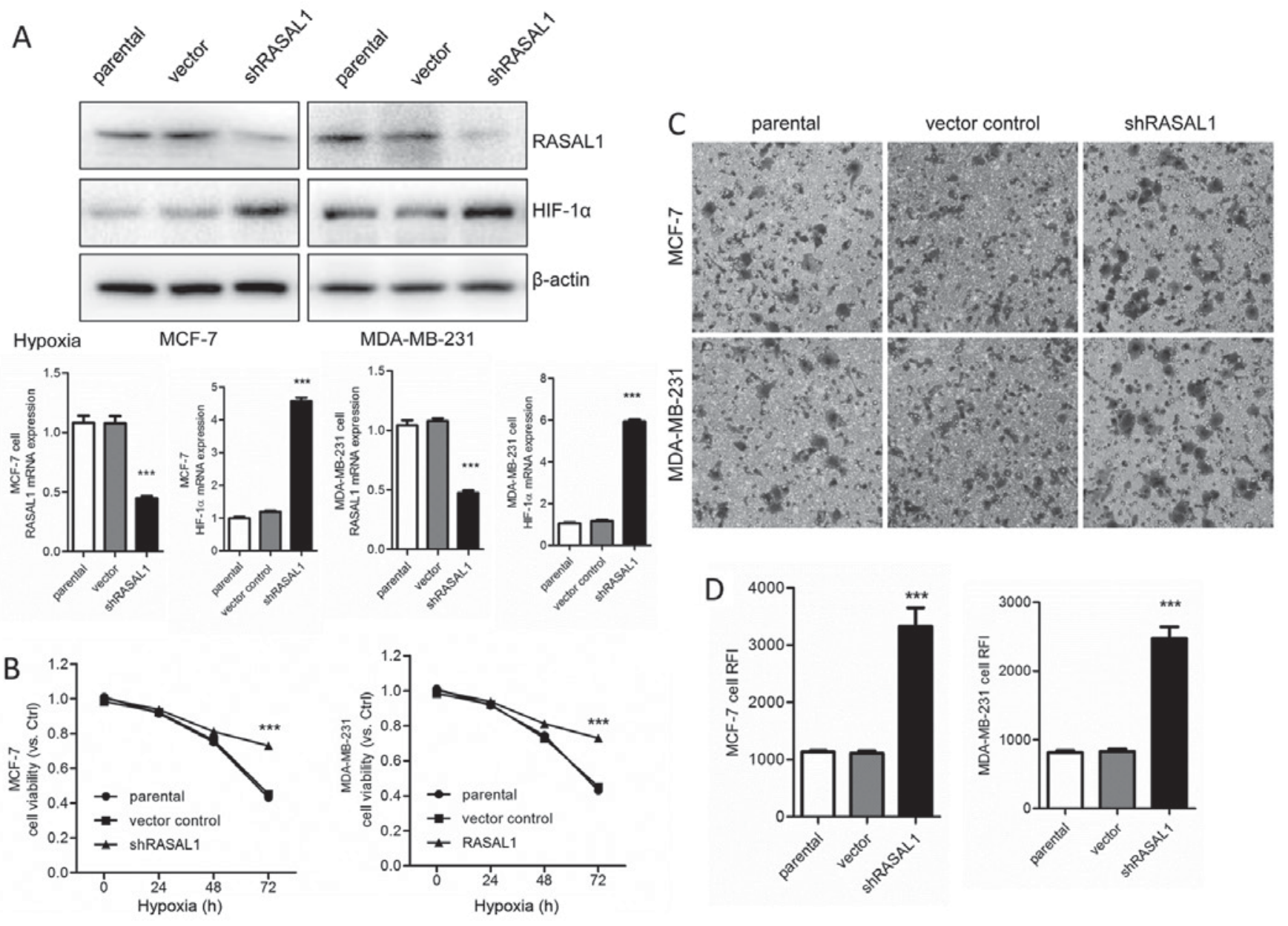

Figure 3. Knockdown of RASAL1 in breast cancer cell lines ameliorates hypoxic cell viability and cell invasion in vitro by increasing the protein levels of HIF-1 $\alpha$ and intracellular levels of ROS. (A) MCF-7 and MDA-MB-231 cells were transfected with RASAL1-specific or scramble shRNA lentivirus for $72 \mathrm{~h}$. The expression of RASAL1 and HIF-1 $\alpha$ mRNA and protein levels were measured by quantitative polymerase chain reaction and western blot analysis (B) Cell viability is shown as the fold change in viability from the normoxic control in MCF-7 and MDA-MB-231 cells treated with hypoxia for various times. (C) Repression of invasion ability following knockdown of RASAL1 in MCF-7 and MDA-MB-231 cells under hypoxia for $24 \mathrm{~h}$ was detected using a Transwell assay (magnification, x100). (D) Intracellular ROS accumulation was detected using a dichloro-dihydro-fluorescein diacetate probe after 24 h of hypoxia treatment. Each bar represents the mean \pm standard error of the mean. The results were reproduced in three independent experiments. ${ }^{* * *} \mathrm{P}<0.001$ vs. the control group. RASAL1, RAS protein activator-like 1; HIF-1 $\alpha$, hypoxia inducible factor-1 $\alpha$; ROS, reactive oxygen species; shRNA, short hairpin RNA; RFI, relative fluorescence intensity.

human cancers; it is also a significant determinant of outcome in numerous human malignancies and is associated with treatment resistance and metastasis $(13,14)$. Hypoxia-induced tumor cell migration may be a major contributor to cancer cell intravasation; the first step of the metastatic cascade. As a result of poor vascularization of the tumor, hypoxia gives rise to significant changes in tumor cell gene expression patterns, metabolism and behavior, which are believed to work in concert to promote tumor cell motility (15). Although great effort has been made, clinically effective therapies targeting hypoxia resistance are still underdeveloped, which may be attributed to the complexity of the development of hypoxia resistance. Thus, in the present study, a hypoxia treatment model of the breast cancer cell lines MCF-7 and MDA-MB-231 was established, which simplified simulation of the process of hypoxic conditions in breast cancer in vitro.

HIF-1 $\alpha$ is induced by intratumoral hypoxia and is involved in crucial aspects of cancer biology, including angiogenesis, cell survival, glucose metabolism and invasion. It has been associated with increased patient mortality in several cancer types (16). In preclinical studies, HIF-1 $\alpha$ inhibition lowers the level of HIF-1 $\alpha$ and the downstream targets and affects tumor vascularization in patients (17). As a result, efforts are underway to identify inhibitors of HIF-1 $\alpha$ and to test their efficacy as anticancer therapeutics. An ever-increasing number of agents are constantly being reported that inhibit HIF-1 $\alpha$ expression and/or activity. Targeting this oncogenic protein may be a potential strategy in the treatment of malignancies.

In the present study, increased expression of HIF-1 $\alpha$ was observed in the hypoxia-treated breast cancer cell lines, MCF-7 and MDA-MB-231, compared with the normoxia group. More significantly, this upregulation was concomitant with increased expression of RASAL1, which is activated by intracellular $\mathrm{Ca}^{2+}$ accumulation and finally attenuation of RAS activity to inhibit tumor growth (18).

These observations implicate that the exogenous expression of RASAL1 in MCF-7 and MDA-MB-231 breast cancer cells may deteriorate its hypoxia resistance in vitro, which is accompanied with decreased expression of HIF-1 $\alpha$ at the mRNA and protein levels. It also inhibited cell migration ability and decreased 

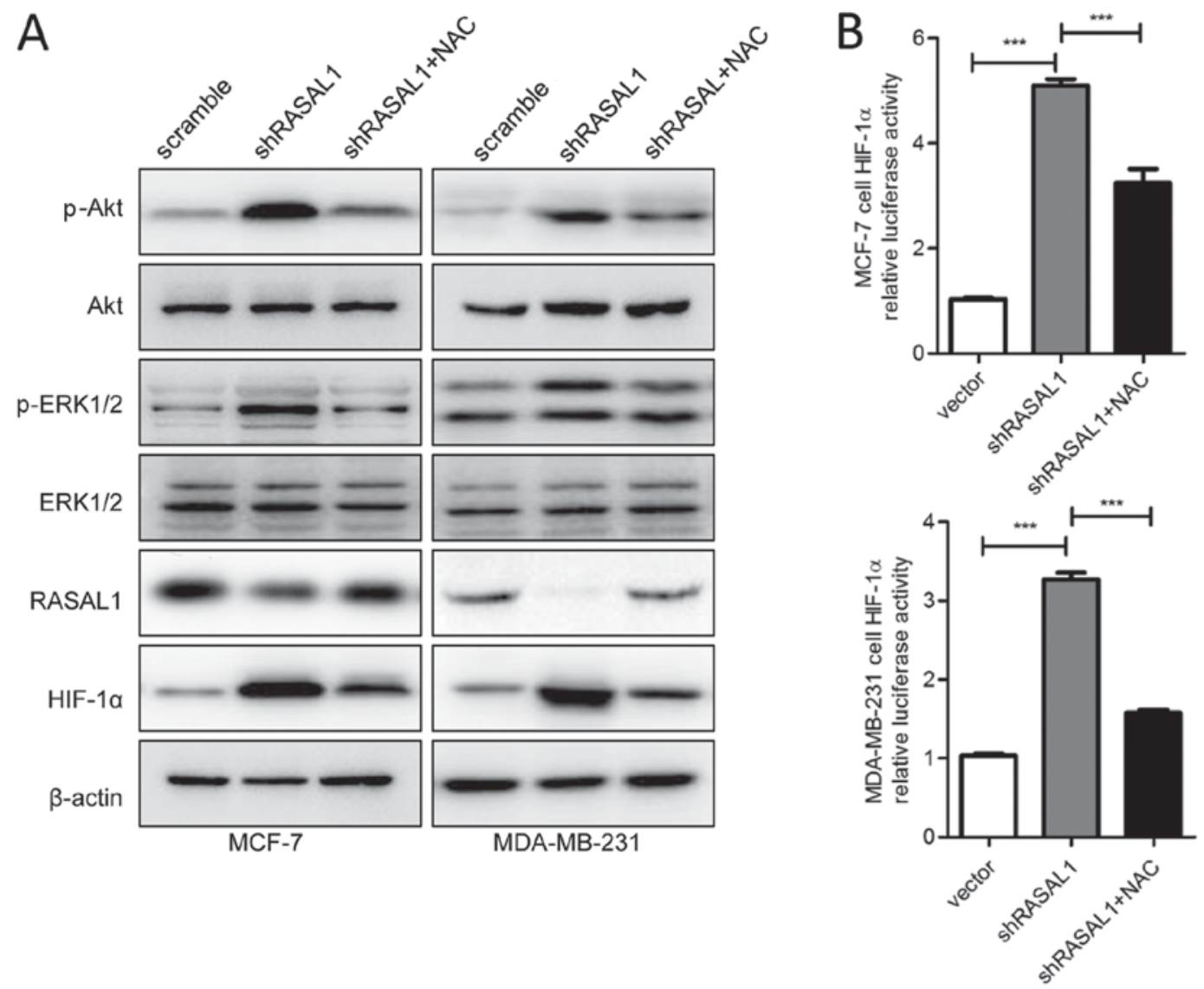

Figure 4. RASAL1 regulates HIF-1 $\alpha$ expression through ROS-ERK/Akt pathway. (A) MCF-7 and MDA-MB-231 cells were transfected with scramble and shRASAL1 lentivirus for $24 \mathrm{~h}$, then treated with $10 \mathrm{mmol} / 1 \mathrm{NAC}$ for $36 \mathrm{~h}$. Total lysates were harvested for the assay of RASAL1, HIF-1 $\alpha$, ERK1/2, Akt, phosphorylated ERK1/2 (P-ERK1/2) and phosphorylated Akt (P-Akt) protein levels using western blot analysis. (B) RASAL1 expression vector increased the luciferase activity of HIF-1 $\alpha$ 3'-UTR by in MCF-7 and MDA-MB-231 cells, while $10 \mathrm{mmol} / 1$ NAC inhibited the effect of shRASAL1. Luciferase activity was determined $60 \mathrm{~h}$ after transfection. Each bar represents the mean \pm standard error of the mean. The results were reproduced in three independent experiments. ${ }^{* * *} \mathrm{P}<0.001$. RASAL1, RAS protein activator-like 1; HIF-1 $\alpha$, hypoxia inducible factor-1 $\alpha$; ROS, reactive oxygen species; ERK, extracellular signal-regulated kinase; shRASAL1, short hairpin RASAL1; NAC, N-acetyl cysteine.

intracellular ROS levels, indicating that RASAL1/HIF-1 $\alpha$ is linked with hypoxia resistance in breast cancer. Furthermore, knockdown of RASAL1 resisted the treatment of hypoxia in MCF-7 and MDA-MB-231 cells, and restored the migration ability and accumulation of ROS levels in vitro. These findings provide direct evidence that RASAL1 may be a critical inducer in the development of hypoxia resistance in breast cancer, which may contribute to tumor progression.

In the present study, western blot analysis was used to further elucidate the molecular mechanism of RASAL1 on the expression of HIF-1 $\alpha$ through the accumulation of ROS levels in breast cancer. It was observed that downregulation of RASAL1 induced the phosphorylation of Akt and ERK1/2 within the MCF-7 and MDA-MB-231 cell lines. Luciferase assay revealed that shRASAL1 significantly upregulated the transactivation of HIF-1 $\alpha$ gene promoters. Additionally, treatment with $10 \mathrm{mmol} / \mathrm{l}$ NAC for $36 \mathrm{~h}$ significantly inhibited the phosphorylation of Akt and ERK1/2 and the transactivation of HIF-1 $\alpha$ gene promoters, suggesting that RASAL1 serves an essential role in hypoxia resistance, which may be mediated via its direct regulation of the expression of HIF-1 $\alpha$ in breast cancer cells, which affects the accumulation of ROS levels in vitro.

Further investigation is required to establish to what extent this RASAL1/HIF-1 $\alpha$ axis functionally aids in promoting the progression of hypoxia resistance in breast cancer. However, the present study provides direct evidence that manipulating the RASAL1/HIF-1 $\alpha$ axis may be a novel strategy for current breast cancer treatment.

\section{References}

1. Youlden DR, Cramb SM, Dunn NA, Muller JM, Pyke CM and Baade PD: The descriptive epidemiology of female breast cancer: An international comparison of screening, incidence, survival and mortality. Cancer Epidemiol 36: 237-248, 2012.

2. Ward C, Langdon SP, Mullen P, Harris AL, Harrison DJ, Supuran CT and Kunkler IH: New strategies for targeting the hypoxic tumour microenvironment in breast cancer. Cancer Treat Rev 39: 171-179, 2013.

3. Lee JW, Bae SH, Jeong JW, Kim SH and Kim KW: Hypoxia-inducible factor (HIF-1) $\alpha$ : Its protein stability and biological functions. Exp Mol Med 36: 1-12, 2004.

4. Ohta M, Seto M, Ijichi H, Miyabayashi K, Kudo Y, Mohri D, Asaoka Y, Tada M, Tanaka Y, Ikenoue T, et al: Decreased expression of the RAS-GTPase activating protein RASAL1 is associated with colorectal tumor progression. Gastroenterology 136: 206-216, 2009.

5. Ngeow J and Eng C: RASAL1 in thyroid cancer: Wisdom from an old foe. J Natl Cancer Inst 105: 1597-1599, 2013.

6. Chen H, Cheng ZY, Pan Y, Wang Z, Liu Y and Zhang JQ: RASAL1 influences the proliferation and invasion of gastric cancer cells by regulating the RAS/ERK signaling pathway. Hum Cell 27: 103-110, 2014. 
7. Kolfschoten IG, van Leeuwen B, Berns K, Mullenders J, Beijersbergen RL, Bernards R, Voorhoeve PM and Agami R: A genetic screen identifies PITX1 as a suppressor of RAS activity and tumorigenicity. Cell 121: 849-858, 2005.

8. Shah T, Krishnamachary B, Wildes F, Mironchik Y and Bhujwalla ZM: Invasion and degradation of extracellular matrix decreases in HIF- $1 \alpha$ and HIF-2 $\alpha$-silenced MDA-MB-231 human breast cancer cells. Cancer Res 72: 3943, 2012.

9. Luo W, Chang R, Zhong J, Pandey A and Semenza GL: Histone demethylase JMJD2C is a coactivator for hypoxia-inducible factor 1 that is required for breast cancer progression. Proc Natl Acad Sci USA 109: E3367-E3376, 2012.

10. Jing Y, Liu LZ, Jiang Y, Zhu Y, Guo NL, Barnett J, Rojanasakul Y, Agani F and Jiang BH: Cadmium increases HIF-1 and VEGF expression through ROS, ERK, and AKT signaling pathways and induces malignant transformation of human bronchial epithelial cells. Toxicol Sci 125: 10-19, 2012.

11. Wu B, Zhang F, Yu M, Zhao P, Ji W, Zhang H, Han J and Niu R: Up-regulation of Anxa2 gene promotes proliferation and invasion of breast cancer MCF-7 cells. Cell Prolif 45: 189-198, 2012.

12. Kitajima Y and Miyazaki K: The critical impact of HIF-1a on gastric cancer biology. Cancers (Basel) 5: 15-26, 2013.
13. Onnis B, Rapisarda A and Melillo G: Development of HIF-1 inhibitors for cancer therapy. J Cell Mol Med 13: 2780-2786, 2009.

14. Milosevic M, Warde P, Ménard C, Chung P, Toi A, Ishkanian A, McLean M, Pintilie M, Sykes J, Gospodarowicz M, et al: Tumor hypoxia predicts biochemical failure following radiotherapy for clinically localized prostate cancer. Clin Cancer Res 18: 2108-2114, 2012.

15. Leong HS and Chambers AF: Hypoxia promotes tumor cell motility via RhoA and ROCK1 signaling pathways. Proc Natl Acad Sci USA 111: 887-888, 2014.

16. Semenza GL: Targeting HIF-1 for cancer therapy. Nat Rev Cancer 3: 721-732, 2003.

17. Meijer TW, Kaanders JH, Span PN and Bussink J: Targeting hypoxia, HIF-1, and tumor glucose metabolism to improve radiotherapy efficacy. Clin Cancer Res 18: 5585-5594, 2012.

18. Liu D, Yang C, Bojdani E, Murugan AK and Xing M: Identification of RASAL1 as a major tumor suppressor gene in thyroid cancer. J Natl Cancer Inst 105: 1617-1627, 2013. 